\title{
Algebraic Reflections on the Vector Concept in Electrocardiography
}

\section{Part III. Representation of the Electric Field}

\author{
Mitsumasa SATO, M.D.
}

Different from the previous 4 chapters dealing with one electric field, the present chapter concerns a set of the fields. The structure of the set of electric fields and its vectorial nature are discussed by refering to its "homomorphic image" or "representation." Here the widely accepted method in practical electrocardiography, the "mean spatial vector" method, is criticized from this point of view.

This chapter may be summarized by a catchy expression ELECTRIC FIELD IS VECTOR, corresponding to the expression in Part I that ELECTROCARDIOGRAM IS VECTOR.

A

FTER studying the structure of one electric field in previous report, ${ }^{20}$ the set of fields must be analysed to clarify the relation between one and another electric fields or between the fields before and after some change of electromotive force of the heart. This chapter was prepared for this purpose.

\section{Chapter 5 .}

\section{Homomorphic Image of the Elegtric Field}

In this chapter, the set $\bar{\Pi}$ of the $n$-dimensional fields is analysed, where the body surface $\overline{\widetilde{S}}$ is considered as the same.

$p, p^{\prime}$ and $p^{\prime \prime}$ are the potentials of a field-position $s$ on $\overline{\mathbb{S}}$ in respective three fields $\overline{\mathfrak{P}}, \overline{\mathfrak{P}}^{\prime}$ and $\overline{\mathfrak{P}}^{\prime \prime}$ in $\bar{\Pi}$. When the relation $p=p^{\prime}+p^{\prime \prime}$ holds for any $s, \overline{\mathfrak{P}}$ may be expressed as the sum of $\overline{\mathfrak{P}}^{\prime}$ and $\overline{\mathfrak{P}}^{\prime \prime}$, and the addition will be defined in $\Pi$. $\left(\bar{P}=\bar{P}^{\prime}+\overline{\mathfrak{P}}^{\prime \prime}\right)$ Similarly, an external multiplication by $F$ is defined in $\bar{\Pi}$, when $p=$ $a p^{\prime}, a \epsilon F$ holds for any $s . \quad\left(\hat{P}=a \bar{P}^{\prime}\right) \quad$ Then, $\bar{\Pi}$ is extended to $\Pi$, the set of all linear combinations of a finite number of fields in $\bar{\Pi}$. $\Pi$ is more concretely impressed, when it is studied in connection with $\Pi$.

As $\mathfrak{i} \in \Pi$ is the set of linear combinations of elements $p^{1}, p^{2}, \ldots \ldots, p^{n}$ corresponding to adequately selected basic field-position $s^{18)} s^{1}, s^{2}, \ldots \ldots, s^{n}$ on $\overline{\mathscr{S}}$, the combination of these $n$-elements, $\bar{P}=\left(\begin{array}{c}p^{1} \\ p^{2} \\ \vdots \\ p^{n}\end{array}\right)$, may represent $\bar{\beta}$. Set $\overline{\mathbf{P}}$ consisting of $\bar{P}$ 's cor-

From the Second Department of Internal Medicine, Faculty of Medicine, University of Tokyo, Tokyo. 
responding to all the fields in $\Pi$ is homomorphic to II with regard to the addition and the external multiplication in $\overline{\mathbf{P}}$, when they are defined in the similar way as in $\Pi$. When the components $p^{1}, p^{2}, \ldots \ldots, p^{n}$ of $\bar{P}$ are mapped to $P^{1}, P^{2}, \ldots \ldots, P^{n}$ by means of mapping- $\pi$ (Chapter 4$), \bar{P}=\left(\begin{array}{c}p^{1} \\ p^{2} \\ \vdots \\ p^{n}\end{array}\right)$ is mapped to $P^{*}=\left(\begin{array}{c}P^{1} \\ P^{2} \\ \vdots \\ P^{n}\end{array}\right)$. Set $\mathrm{P}^{*}$ consisting of $P^{*}$ 's corresponding to all $\bar{P}$ in $\overline{\mathbf{P}}$ is homomorphic to $\overline{\mathbf{P}}$. And as $\overline{\mathbf{P}}$ is homomorphic to $\Pi, \mathbf{P}^{*}$ is homomorphic to $\Pi$. And as

$$
P^{*}=\left(\begin{array}{c}
P^{1} \\
P^{2} \\
\vdots \\
P^{n}
\end{array}\right)=\left(\begin{array}{c}
p_{1}^{1} p_{2}^{1} \cdots \cdots \cdots \cdots \cdots \\
p_{1}^{2} p_{2}^{2} \cdots \cdots \cdots \cdots \cdots \\
\cdots \cdots \cdots \cdots \cdots \cdots \cdots \\
\cdots \cdots \cdots \cdots \cdots \\
p_{1}^{n} p_{2}^{n} \cdots \cdots \cdots \cdots \cdots \\
p_{n}^{n}
\end{array}\right)=(P)
$$

$\mathbf{P}^{*}$ is a linear algebra composed of $n \times n$ matrices. ${ }^{1,4)}$ Hence,

1) The set of the equi-dimensional electric fields has a linear algebra composed of $n \times n$ matrices as its homomorphic image, where $n$ is dimension-number of the fields.

2) The set of the fields has a finite-dimensional vector space as its homomorphic image.

3) The set of the field is represented by the algebra composed of $n \times n$ matrices. In the sense of 2), or in the homomorphic sense,

" the electric field may be regarded as a vector." ELECTRIC Field is Vector.

It was mentioned in Chapter 2 that an electrocardiogram recorded from a point on the body surface might be regarded as a vector. But the author found in the above 2) that the set of electrocardiograms recorded from all over the body surface, the electric field itself, might also be regarded as a vector in the homomorphic sense. In practical electrocardiography, the concept "mean spatial vector" has been widely used recently. The author's opinion about utility of this concept is that the mean spatial vector has been introduced in order to "compare" several fields with each other. Every field is represented by its own mean spatial vector, and comparison of the vectors representing the respective fields makes that of the fields possible. In this sense, a field may have been regarded as a vector. The results above-mentioned, 1), 2) and 3), correspond to this conventional view. But it is still unknown, how and to what degree the mean vector, integration of instant vector, could represent the field, and what relationship have the vectors, representing the fields, between each other. In order to solve these questions, the author is going to discuss generalizing the problem of representation of the field $\Pi$ in this chapter.

The "representation" here is introduced for practical purposes and has somewhat different sense from that in algebra, ${ }^{1,2)}$ although this concept covers the representation in algebra. There are a set $S$ and another set $A$ for which a law of composition $\omega$ is defined. When an element of $S$ corresponds surely to one element of $\boldsymbol{A}$ by correspondence $\gamma$, it is called that " $S$ is represented by $\gamma$ in $A$ ", that an element $a$ of $A$ corresponding to an element $s$ of $S$ is "representation of $s$ by $\gamma$ ", that set $A^{\prime}$ consisting of $a$ 's corresponding to all $s^{\prime}$ ' of $S$ is "representation 
of $S$ by $\gamma$ ", and that " $\gamma$ is a representor of $S$." Of course $A^{\prime} \subseteq A$. When $s_{1}$, $s_{2} E S$ and they have the same representation by $\gamma$, this relation is expressed as $s_{1} \equiv s_{2}(\gamma)$, which is an equivalence relation ${ }^{1,3)}$. Then, a classification of $S$ is possible by this relation. This is said as the "classification of $S$ by its representation $A^{\prime \prime \prime}$. If $S$ has two representations $A_{1}$ and $A_{2}$ in $A$, and if $A_{2} \subset A_{2}$ holds, the representation $A_{1}$ is said "more strict" than $A_{2}$. This is the concept that expresses to some extent the degree of fidelity of the representation.

When one intends to represent $S$ in $A, A$ may be selected in many ways theoretically, but in practice $A$ must have somewhat concrete significance according to the purposes. Moreover, the law of composition $\omega$ in $A$ may be expected to have a concrete significance, because $\omega$ is the law of composition also in $S$ when it is mapped homomorphically into $S$, though $S$ has originally no law of composition. If there are defined m-practically significant laws of composition in $A, A^{\prime}$ is a "representation of $m$-degrees". The more strict is the representation, the more it is precise. And the higher is its degree, the more it is practically significant.

Here is a familiar example of representation. $S$ is here a set of all the points on a two-dimensional plane (Fig. 4). From a point $s$ in $S$, perpendicular line is drawn to a line $x$, and its foot is signed as $s_{x}$. The set consisting of $a_{x}$ 's corresponding to all s's in $S$ is $F$ (the field of real numbers), where $a_{x}$ is a distance from a fixed point $O$ on $x$ to $s_{x}$, measured by certain unit. Then, $F$ is a representation of $S$ or $S$ is represented in $F$ and by $F\left(F=A_{1}\right)$. Representation $a_{x}$ of point $s$ signifies a sort of distance from $O$ to $s$. When two laws of composition $\omega_{1}$ and $\omega_{2}$, are defined in $A_{1}$ as $a_{1 x} \omega_{1} a_{2 x}=a_{1 x}-a_{2 x}, a_{x 1} \omega_{2} a_{2 x}=a_{1 x} / a_{2 x}$,they are significant. $\omega_{1}$ signifies a sort of distance between two points $s_{1}$ and $s_{2}$, and $\omega_{2}$ signifies a ratio of

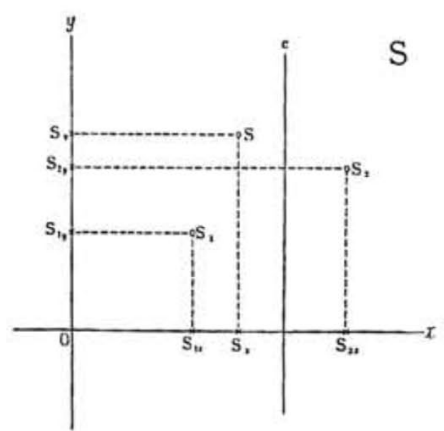

Fig. 1. An example of representation.

distances from $O$ to $s_{1}$ and $s_{2}$. There may be thought all kinds of laws of composition other than $\omega_{1}$ and $\omega_{2}$, but they have lesser significance than $\omega_{1}$ and $\omega_{2}$, in the usual sense. Thus, $A_{1}$ is a representation of two degrees.

A more strict representation of $S$ than $A_{1}$, shall be demonstrated as follows. A line $y$, perpendicular to $x$, crosses $x$ at $O . s_{y}$ and $a_{y}$ are determined in the same way as $s_{x}$ and $a_{x}$. Set consisting of $a_{y}$ 's is $F$. If addition and multiplication are defined in $A_{2}$, the set of ordered sets $\left(a_{x}, a_{y}\right)$ 's of two real numbers $a_{x}$ and $a_{y}$, as $\left(a_{x}, a_{y}\right)+\left(a_{x}^{\prime}, a_{y}^{\prime}\right)=\left(a_{x}+a_{x}^{\prime}, a_{y}+a_{y}^{\prime}\right),\left(a_{x}, a_{y}\right) \cdot\left(a_{x}^{\prime}, a_{y}^{\prime}\right)=\left(a_{x} \cdot a_{x}^{\prime}, a_{y} \cdot a_{y}^{\prime}\right), A_{2}$ is also a field. Here, we have a representation of $S$ by making the element $\left(a_{x}, a_{y}\right)$ of $\boldsymbol{A}_{2}$ correspond to point $s$. Representation $\left(a_{x}, a_{y}\right)$ of $s$ signifies a sort of quantity which shows how the point $s$ is distant from the point $O$. When two laws of 
composition $\omega_{1}$ and $\omega_{2}$ are defined in $A_{2}$ as $\left(a_{1 x}, a_{1 y}\right) \omega_{1}\left(a_{2 x}, a_{2 y}\right)=\left(a_{1 x}, a_{1 y}\right)-\left(a_{2 x}, a_{2 y}\right)$, $\left(a_{1 x}, a_{1 y}\right) \omega_{2}\left(a_{2 x}, a_{2 y}\right)=\left(a_{1 x}, a_{1 y}\right) /\left(a_{2 x}, a_{2 y}\right)$, they are also significant in almost the same meaning with $\omega_{1}$ and $\omega_{2}$ in $\boldsymbol{A}_{1}$. Thus, $\boldsymbol{A}_{2}$ is a representation of two degrees. And as obviously $A_{1} \subset A_{2}$ holds, $A_{2}$ is more strict than $A_{1}$.

A class in the classification by $A_{1}$ shows a straight line $c$, any point on which has the same representation in $A_{1}$. And a class in the classification by $A_{2}$ is a only one point. Hence, as to this example, there is no more strict representation than $\boldsymbol{A}_{2}$.

$\Pi$ is represented in $P^{*}$, and by $P^{*}$. In this case, $P^{*}$ is a representation in also algebraic meaning. The character and the meaning of the representation $\boldsymbol{P}^{*}$ of $\Pi$ shall be discussed as follows. This will clarify the character and structure of $\Pi$ and $\bar{I}$.

When $\overline{\mathfrak{P}}$ and $\bar{D}$ in $\bar{\Pi}$ have the same representation in $P^{*}$ or $\overline{\mathfrak{P}}$ and $\overline{\mathscr{Q}}$ are in the same class in the classification by $P^{*}$, the bases $P$ and $Q$ of potentials $p$ and $q(p \in \overline{\mathfrak{P}}, q \in \overline{\mathfrak{Q}})$ are expressed as $P=\left(P^{\prime}\right) A, \quad Q=\left(Q^{\prime}\right) B$ from formula $(2-13)^{\prime \prime}$. As $(P)=(Q)$ here, or in other words, as the bases of the two fields are equal, $P=Q$ or $p_{i}=q_{i}(i=1,2, \ldots \ldots, n)$ holds, if $A=B$, where $P=\left(p_{1}, p_{2}, \ldots \ldots, p_{n}\right)$, $Q=\left(q_{1}, q_{2}, \ldots \ldots, q_{n}\right)$. Hence, " not only the bases of two electric fields in the same class, but also the bases of electrocardiograms of the respective fields are equal, if field-positioncoordinates of the two electrocardiograms are equal".

One of the laws of composition, $\omega_{1}$, in $P^{*}$ is defined as $Q^{*} \omega_{1} P^{*}=Q^{*} / P^{*}=$ $\left(Q P^{-1}\right)=(K) \quad|P| \neq 0$, where $P^{*}$ and $Q^{*}$ are the representation of $\bar{P}$ and $\bar{\Omega}$, respectively. The matrix $(K)$ is called sometimes as the "ratio of the two fields", or as the "field angle from $\overline{\mathfrak{P}}$ to $\bar{\Omega}$ ". ${ }^{11,177,18)}$ The significance of this law of composition will be discussed as follows.

When the extensions of two fields $\overline{\mathfrak{P}}$ and $\overline{\mathbb{Q}}$ in $\Pi$ are equal, that is, when $[\overline{\mathfrak{P}}]=[\overline{\mathrm{D}}]$

$$
\text { or } \quad\left[p^{1}, p^{2}, \ldots \ldots p^{n}\right]=\left[q^{1}, q^{2}, \ldots \ldots, q^{n}\right]
$$

holds, an equivalence relation such as $\overline{\mathfrak{P}} \equiv \overline{\mathcal{L}}(f)$ holds. Then, $\Pi$ is classified by the relation $(f)$, and a class in this classification is named particularly as a "family"18) in order to differentiate from the "class" in the classification by representation $P^{*}$ (Fig. 5). When $P^{*}$ and $Q^{*}$ are the representations of respective two electric fields $\overline{\mathfrak{B}}$ and $\bar{\Omega}$ in the same family, $Q^{*} / P^{*}=(K)$,

$$
\therefore \quad(Q)=(K P)
$$

From formula $(5-2), \quad \bar{Q}=(\mathcal{F}) \overline{\boldsymbol{P}} \quad|\mathcal{J}| \neq 0$ holds. $\therefore(Q)=(\mathcal{J} P)$

From formula $(5-3)$ and $(5-4), \quad(\mathcal{J})=(K) \quad \therefore \quad \bar{Q}=(K) \bar{P}$

And from formula $(2-13)^{\prime}, \quad p=A \cdot \bar{P}, q=B \cdot \bar{Q} \quad\left(p \in \overline{P_{\beta}}, p \in \overline{\mathbb{L}}\right), \quad \therefore \quad q=$ $B \cdot \bar{Q}=B \cdot(K) \overline{\boldsymbol{P}}=\left(K^{\prime}\right) B \cdot \overline{\boldsymbol{P}} \quad$ Hence, $p=q$, if

"Two electrocardiograms of respective two fields in the same family are equal, if the relationship (5-6) holds between field-position-coordinates of the respective two electrocardiograms."

To say in other words,

"The ratio of the two fields, $(K)=Q^{*} / P^{*}$, may be regarded as a required quantity (transformation) in order to eliminate the "difference" between them."117),18)

This is the practical significance of the law of composition $\omega_{1}$ defind in the 
representation $P^{*}$ of the electric fields. A more practical explanation of the field angle was reported before. ${ }^{18)}$

If $\bar{P}$ and $\overline{\mathfrak{D}}$ are not in the same family, the field $\overline{\mathbb{H}^{i}}$ could be found in the same family with $\bar{q}$ (Fig. 5), and $R^{*} / P^{*}=Q^{*} / P^{*}=(K)$.

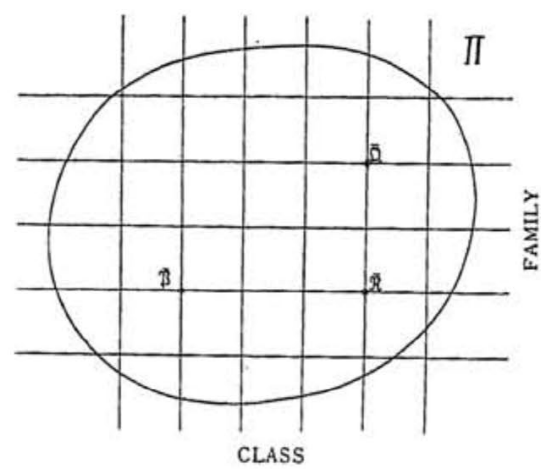

Fig. 2. Two sorts of classifications of the electric fields. The one is by representation (class), and the other is by extension (family).

Hence, if the relation (5-8) between field-position-coordinates $A$ and $C$ of $p$ and $r(p \in \overline{\mathfrak{P}}, r \in \overline{\mathfrak{P}})$, respectively, $C=\left(K^{\prime-1}\right) A$

holds, then $p=r, \quad \therefore \quad P=R$

And as $Q^{*}=R^{*}$ from formula (5-7), $\overline{\mathbb{Q}}$ and $\overline{\mathfrak{R}}$ are in the same class. When $B$ is field-position-coordinate of $q \in \bar{Q}$, and when $Q$ and $R$ are the bases of $q$ and $r$, then, $Q=R$ holds if $B=C$. Hence, from formula (5-8) and (5-9), $P=Q$ holds if $B=\left(K^{-1}\right) A$.

"At least, the bases of two electrocardiograms of respective two fields are equal, if the relation (5-6) holds between field-position-coordinates of the two electrocardiograms."

The above is a discussion concerning $\omega_{1}$ but another law of composition $\omega_{2}$ defined as an addition, may be practically significant, although this is not so important here and omitted. Then,

\section{" $P^{*}$ is a representation of $\Pi$, of two degrees."}

It was mentioned that, in practical electrocardiography, the electric field had usually been represented by mean vector such as Einthoven's two-dimensional mean vector (frontal plane vector) and recently as spatial mean vector. According to our terminology in this chapter, $\Pi$ has been represented in a two- or three-dimensional vector space. When representation of $\Pi$ in three-dimensional vector space $V_{3}$ is $V^{\prime}, \quad V^{\prime} \subseteq V_{3}$ hols naturally. As $V_{3} \subset V_{n}$, where $n$ is the dimension-number of the fields in $\bar{\Pi}, V^{\prime} \subset V_{n}$. And as obviously $V_{n} \subset P^{*}, \therefore V^{\prime} \subset P^{*}$. Hence,

"The representation of $\overline{\bar{\Pi}}$ by the method of mean spatial vector is less strict than that by $P^{*}$." or, "The representation of $\bar{\Pi}$ by $P^{*}$ is more strict than that by the method of mean spatial vector."

As to the significant laws of composition in $V_{2}$ or $V_{3}$, the two-addition and angle $\hat{A}_{P Q}$ between the mean vectors $V_{P}$ and $V_{Q}$ representing respectively $\overline{P_{P}}$ and $\bar{D}$ in $\bar{\Pi}$-are given usually. Thus, the respresentation $V^{\prime}$ is of two-degrees. The angle $\hat{A}_{P Q}$ is the law of composition which corresponds to the ratio of two fields $(K)$ in our representation $P^{*} . \hat{A}_{P Q}$ is determined uniquely for given two vectors 
$V_{P}$ and $V_{Q}$, but conversely, $V_{Q}$ is not determined even if $V_{P}$ and $\hat{A}_{P Q}$ are given. For this reason, it may be better theoretically to give, as an law of composition $\omega_{2}$, subtraction $V_{P} \omega V_{Q}=V_{P}-V_{Q}$ instead of the angle $\hat{A}_{P Q}$, if the practical point is not taken into consideration

\section{Summary and Conclusion}

(1) It is the author's opinion that the method of "mean spatial vector" has been introduced in order to compare several fields with each other and it has been efficient and available for that purpose. Every field is represented by its own mean spatial vector, and comparison of the vectors would make that of the fields possible. But it has been still unknown, how and to what degree the mean vector could represent the field, and what relation have the vectors representing the fields between each other. This problem of representation was generalized in this chapter.

(2) The set of equi-dimensional electric fields is represented by liner algebra composed of $n \times n$ matrices, when $n$ is the dimension-number of the field. The algebra is a representation of the fields.

(3) Thus, the set of the fields has a finite-dimensional vector space as its homomorphic image.

(4) Hence, a field may be regarded as a vector in the homomorphic sense. ELECTRIC FIELD IS VECTOR.

(5) When the field is represented by its own mean spatial vector, the field is regarded obviously as a vector. The above (4) is the result corresponding to this conventional view,

(6) The practical significance of law of composition in the representation was discussed, particularly the concept "ratio of two fields or field angle" was explained. The ratio of two fields may be considered as a required quantity (transformation) in order to eliminate the "difference" between these two, and may correspond to the angle between two mean spatial vectors of the respective two fields.

(7) The concept "more strict" representation was defined, and the degree of fidelity of the representation was taken into consideration. It was demonstrated that the representation by the algebra in (2) was more strict than that by the method of mean spatial vector.

(8) As to the application of the representation by the algebra, the author refers to their last reports. ${ }^{17), 18}$

\section{REFERENCES}

1. Birkhoff, G. and Maclane, S.: A Survey of Modern Algebra, The Macmillan Co., New York, 1941.

2. Queysanne, M. et Delachet, A.: Algèbre Moderne, Presses Universitaires de France (Collection Que Sais-Je? N ${ }^{\circ} 661$ ), 1955. 
3. Shoda, K.: Text-book of Algebra, The Kyoritsu Pub. Co., Tokyo, 1946 (In Japanese).

4. Shoda, K.: An Outline of Algebra, The Kyoritsu Pub. Co., Tokyo, 1956 (In Japanese).

5. Kono, I.: Theory of Topological Space, The Kyoritsu Pub. Co., Tokyo, 1957 (In Japanese).

6. Wilson, F. N., Rosenbaum, F.F., and Johnston, F.D.: Advances in Int. Med., 2: 1, Interscience Pub., New York, 1947.

7. Goldberger, E.: Unipolar Lead Electrocardiography, Lead and Febiger, Philadelphia, 1955.

8. Frank, E.: Circulation 11: 937, 1955.

9. Burger, H.C. and van Milaan, J.B.: Brit. Heart J. 8: 157; 9: 154; 10 : 229, 1948.

10. Schmidt, O.H., Levine, R. B., Simonson, E., and Dahl, J. : Am. Heart J. 45: 416, 500 and 655, 1953.

11. Grishman, A. and Sherlis, L.: Spatial Vectorcardiography, W. B. Saunders, Philadelphia, 1952.

12. Duchosal, P.W. and Sulzer, R. : La Véctocardiographie, S. Karger, Basel, 1949.

13. Grant, R. P.: Circulation 1: 878, 1950.

14. Grant, R.P. and Estes, E.H.: Spatial Vector Electrocardiography, The Blakiston Co., Philadelphia, 1952.

15. Milnor, W. R., Talbot, S. A., and Newman, E. V.: Circulation 7: 545, 1953.

16. Frank, E.: Am. Heart J. 47: 757, 1954.

17. Sato, M. and Murao, S.: Naika 4: 849, 1959 (In Japanese).

18. Sato, M. : Jap. Circulation J. 23: 1271, 1959.

19. Sato, M. : Jap. Heart J. 1: 317, 1960.

20. Sato, M. : Jap. Heart J. 1: 432, 1960. 